\title{
Myelomeningocele with Unilateral Right Renal Agenesis: A Case Report
}

\author{
Hajime Maeda, MD ${ }^{1}$ Hayato Go, MD, $\mathrm{PhD}^{1} \quad$ Jun Sakuma, MD, $\mathrm{PhD}^{2}$ Takashi Imamura, MD, $\mathrm{PhD}^{1}$ \\ Maki Sato, MD, PhD ${ }^{1}$ Nobuo Momoi, MD, $\mathrm{PhD}^{1}$ Mitsuaki Hosoya, MD, $\mathrm{PhD}^{1}$ \\ ${ }^{1}$ Department of Pediatrics, Fukushima Medical University School of \\ Medicine, Fukushima, Fukushima, Japan \\ 2 Department of Neurosurgery, Fukushima Medical University School \\ of Medicine, Fukushima, Fukushima, Japan \\ Address for correspondence Hajime Maeda, MD, Department of \\ Pediatrics, Fukushima Medical University School of Medicine, \\ 1 Hikarigaoka, Fukushima City, Fukushima 960-1295, Japan \\ (e-mail: hmaeda@fmu.ac.jp).
}

Am J Perinatol Rep 2018;8:e1-e3.

\begin{abstract}
Keywords

- Chiari malformation

- congenital anomalies

- genitourinary system

- myelomeningocele

- neural tube defects

Congenital anomalies of the spine may occur with malformations of the central nervous, cardiovascular, gastrointestinal, respiratory, and genitourinary systems. This is a case of myelomeningocele with unilateral right renal agenesis in a newborn. The patient suffered complications of cerebrospinal fluid leak and meningitis, but was successfully treated and discharged on day 86 . In this case, unilateral right renal agenesis represented a significant surgical risk because failure of the remaining kidney could result in renal failure. Because congenital anomalies of the spine may be associated with malformations of the genitourinary system, and additional surgeries were necessary in our case following birth, it is very important that the presence of genitourinary malformations be evaluated.
\end{abstract}

The worldwide incidence of neural tube defects ranges from 1 to 10 per 1,000 births with similar frequencies of anencephaly and spina bifida. ${ }^{1}$ Congenital anomalies of the spine have been associated with malformations of the central nervous, cardiovascular, gastrointestinal, respiratory, and genitourinary systems. ${ }^{2}$ We report a case of myelomeningocele with unilateral right renal agenesis.

\section{Case Report}

The mother was a 31-year-old woman (gravida 3 para 3), who had not taken any medications or supplements or had poor antenatal care follow-up. Fetal ventricular enlargement was detected at 35 weeks of gestation, and the baby was delivered by cesarean section at 38 weeks of gestation following detection of a Chiari malformation and myelomeningocele by fetal echography and magnetic resonance imaging (MRI). The baby was a female and weighed 2,368 $\mathrm{g}$ with an Appearance, Pulse, Grimace, Activity, and Respiration (APGAR) score of 5 at 1 minute and 6 at 5 minutes. Endotracheal intubation and mechanical ventilation were initiated shortly after birth because of respiratory distress. The anterior fontanelle was bulged at birth, and a large skin defect and myelomeningocele (the defect, $10 \mathrm{~cm} \times 5 \mathrm{~cm}$ ) were present on her back ( - Fig. 1A). She had no motor power in her lower extremities and had a right congenital talipes equinovarus deformity (-Fig. 1B). Laboratory data were normal. Serum creatinine continued to be elevated until day 2 and spontaneously decreased afterward (-Fig. 2). Chromosomal analysis was normal. Computed tomography of the head and brain and spinal MRI revealed descent of not only the cerebellar tonsil but also the structure of the posterior fossa toward the spinal canal and small post fossa, consistent with Chiari II malformation. Kyphosis was evident. It was difficult to identify the spinal cord in the thoracolumbosacral region, and the right kidney was aplastic received

September 29, 2017

accepted

November 19, 2017
DOI https://doi.org/

10.1055/s-0037-1615818. ISSN 2157-6998.
Copyright $\odot 2018$ by Thieme Medical Publishers, Inc., 333 Seventh Avenue, New York, NY 10001, USA. Tel: +1(212) 584-4662.
License terms

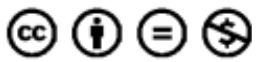




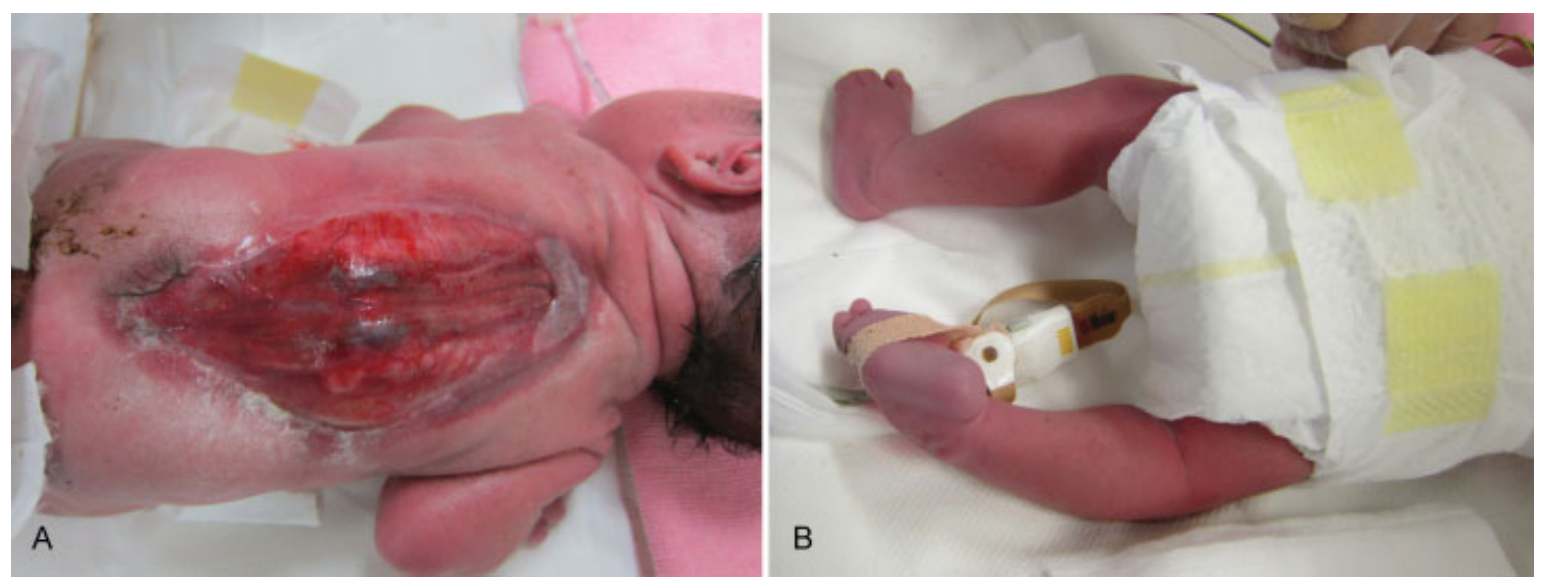

Fig. 1 Physical findings on admission. At birth, (A) the infant had a large skin defect with a myelomeningocele (the defect, $10 \mathrm{~cm} \times 5 \mathrm{~cm}$ ) on her back, and (B) she had no motor power in her lower extremities and had a right congenital talipes equinovarus deformity.

(-Figs. 3A and B). Myelomeningocele repair was performed on day 0 , with cerebrospinal fluid drainage, placing an Ommaya reservoir, and carrying out a bilateral bipedicle flap transfer. On day 12, the patient was extubated. On day 12 , a cerebrospinal fluid leak developed; antibiotics were initiated. On day 23, meningitis was diagnosed from the cerebrospinal fluid. On day 24 , the patient developed apnea and endotracheal intubation and mechanical ventilation were initiated again. On day 29, the reservoir was removed with ventricular drainage. On day 32 , the patient was extubated. Because of occlusion of the external ventricular drainage tube, an Ommaya reservoir was placed again on day 55, and following confirmation that the cerebrospinal fluid was not infected, the reservoir was removed and a ventriculoperitoneal shunt was inserted on day 76 . The patient was discharged on day 86.

\section{Discussion}

The risk of neural tube defects has been associated with socioeconomic status, parental education, maternal/paternal age and occupation, and maternal reproductive history, including country of birth and conception, hyperthermia

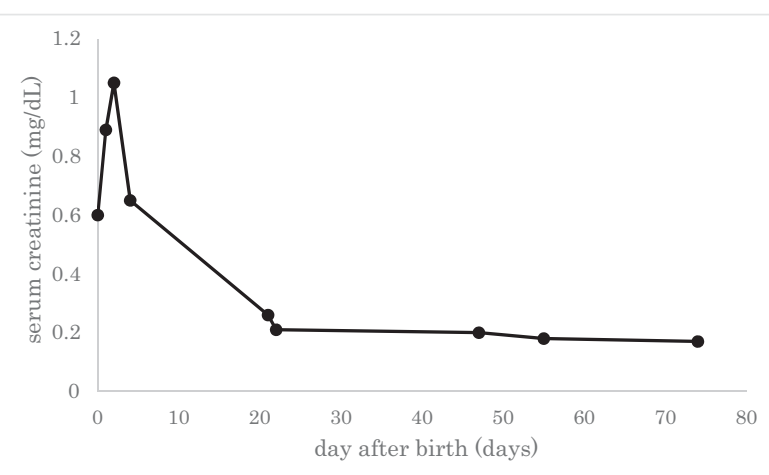

Fig. 2 Serum creatinine from birth until discharge. Serum creatinine value was $0.60 \mathrm{mg} / \mathrm{dL}$ at birth and reached a maximum value $1.05 \mathrm{mg} / \mathrm{dL}$ on day 2 , and decreased afterward. during early pregnancy, hyperglycemia or diabetic obesity, and maternal use of caffeine and medications during early pregnancy. ${ }^{1}$ Normal maternal folate status is important for neural tube closure during embryogenesis, and increasing folate level, significantly reduces the occurrence and recurrence of neural tube defect. ${ }^{3}$ The patient's mother took no medication or supplements during pregnancy and had a low socioeconomic status, all of which were consistent with low maternal folate status.

Congenital anomalies of the spine have also been associated with malformations of the genitourinary system. Whitaker and Hunt reported that 17 of 190 patients with neural tube defects were complicated by congenital renal anomalies, including three with renal agenesis. ${ }^{4}$ Torre et al reported that 3 of 283 cases of meningomyelocele were complicated by unilateral renal agenesis. ${ }^{5}$ The genitourinary system and vertebral column are both of mesodermal origin and develop during the same period of embryonic life. During week 5 of embryogenesis, the vertebral bodies begin to develop from the notochord, and the ureteral buds migrate to the metanephrogenic blastema. Embryonic injury during that stage of development can cause defects in the vertebral column and abnormalities of the kidneys and the urine collection system. ${ }^{6}$ There have been few reports of myelomeningocele with unilateral renal agenesis. These two malformations may either follow different embryonic insults, i.e., be a two-hit occurrence like the two-hit theory of cancer, or the renal event may simply be a rare complication. Myelomeningocele patients with untreated urological complications exhibit vesicouteral reflux and kidney damage. ${ }^{7}$ Our patient did not have kidney damage, but renal agenesis can result in failure of the remaining kidney in response to external injury, inflammation, renal calculus, or tumors. ${ }^{8}$ Therefore, renal agenesis represents a significant surgical risk. Because additional surgeries were necessary in our case, it is important to promptly identify malformations in the genitourinary system. The renal function of the patient is being followed carefully after discharge. 

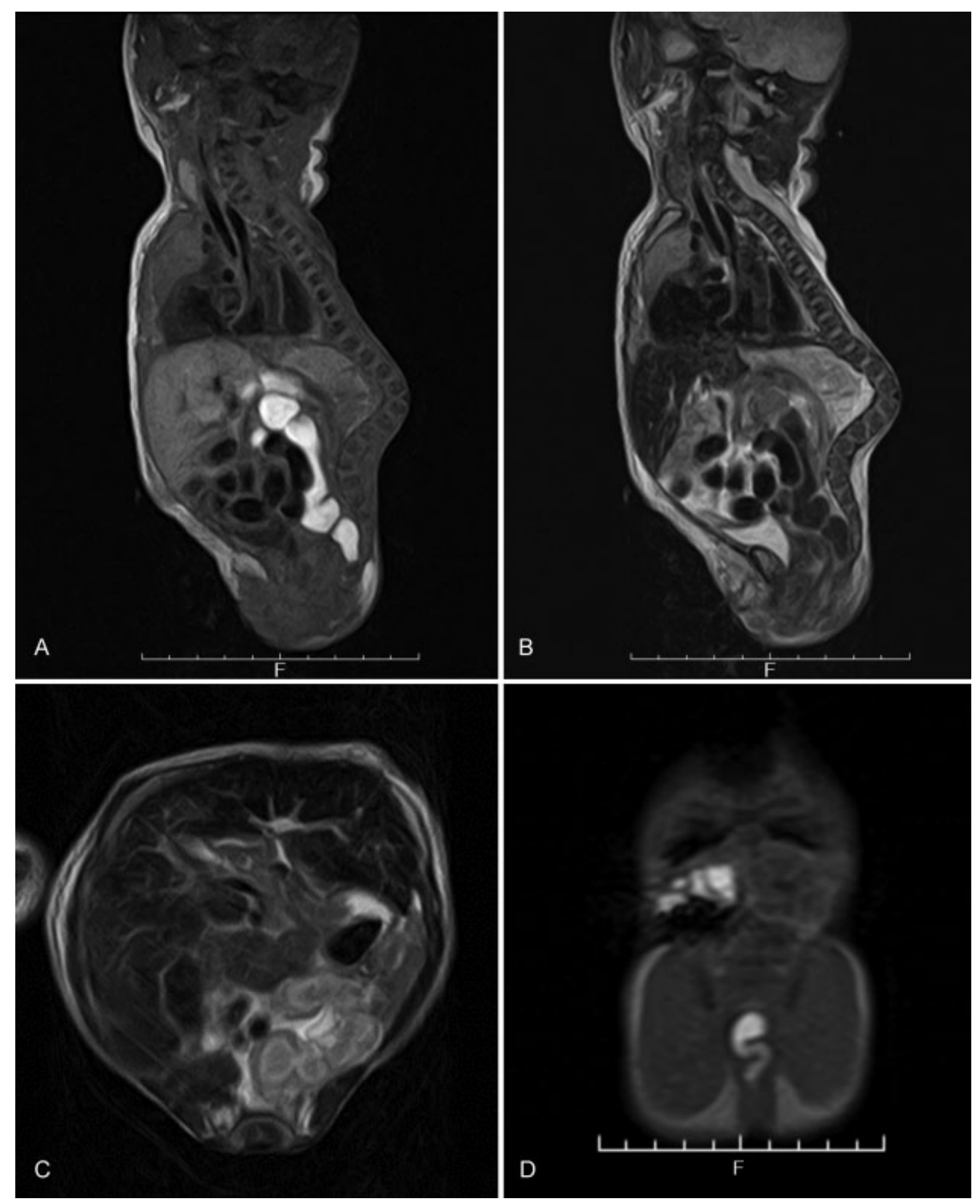

Fig. 3 Magnetic resonance imaging (MRI) on admission. Brain and spinal MRI revealed T1-weighted sagittal (A) descent of the cerebellar tonsil and the structure of the posterior fossa into the spinal canal, and small post fossa, consistent with Chiari ll malformation. Kyphosis was evident. T2-weighted sagittal (B) difficulty in identifying the spinal cord in the thoracolumbosacral region, and T2-weighted axial (C) and T1-weighted coronal (D) an aplastic right kidney.

\section{Conflict of Interest}

None.

\section{References}

1 Au KS, Ashley-Koch A, Northrup H. Epidemiologic and genetic aspects of spina bifida and other neural tube defects. Dev Disabil Res Rev 2010;16(01):6-15

2 Tori JA, Dickson JH. Association of congenital anomalies of the spine and kidneys. Clin Orthop Relat Res 1980;(148):259-262

3 Czeizel AE, Dudás I. Prevention of the first occurrence of neural-tube defects by periconceptional vitamin supplementation. N Engl J Med 1992;327(26):1832-1835
4 Whitaker RH, Hunt GM. Incidence and distribution of renal anomalies in patients with neural tube defects. Eur Urol 1987; 13(05):322-323

5 Torre M, Guida E, Bisio G, et al. Risk factors for renal function impairment in a series of 502 patients born with spinal dysraphisms. J Pediatr Urol 2011;7(01):39-43

6 Vitko RJ, Cass AS, Winter RB. Anomalies of the genitourinary tract associated with congenital scoliosis and congenital kyphosis. J Urol 1972;108(04):655-659

7 Bruschini H, Almeida FG, Srougi M. Upper and lower urinary tract evaluation of 104 patients with myelomeningocele without adequate urological management. World J Urol 2006;24(02):224-228

8 MacEwen GD, Winter RB, Hardy JH. Evaluation of kidney anomalies in congenital scoliosis. J Bone Joint Surg Am 1972;54(07):1451-1454 